\title{
Social safeness and disordered eating: Exploring underlying mechanisms of body appreciation and inflexible eating
}

\author{
Catarina Pinto, MSc \\ Cláudia Ferreira, PhD \\ Ana Laura Mendes, MSc \\ Inês A. Trindade, Ph.D. Student
}

CINEICC

University of Coimbra, Portugal

Correspondence concerning this article should be addressed to:

Ana Laura Mendes, University of Coimbra- Faculty of Psychology and Educational

Sciences, Rua do Colégio Novo, Apartado 6153, 3001-802. Coimbra (Portugal)

E-mail: analauramendes@live.com.pt

Phone: (+351)239851450; (+351)203851462 


\section{Abstract}

Feelings of social safeness and connectedness have been associated with adaptive emotion regulation processes and well-being indicators. Further, literature has demonstrated that interpersonal experiences play an important role in the etiology and maintenance of body and eating psychopathology. However, the study of the role of social variables and emotion regulation processes in the engagement in inflexible eating rules and eating psychopathology is still in its early stages. The current study aims to fill some gaps within the literature and explore the mediator role of body appreciation and inflexible eating rules in the link between social safeness and disordered eating.

Participants were 253 women, aged between 18 and 50 years old, who completed a series of online self-report measures.

Results from the tested path analysis model showed that social safeness holds a significant effect on eating psychopathology, through the mechanisms of body appreciation and inflexible eating rules. Also, results suggested that women who present higher levels of social safeness tend to present a more positive and respectful attitude towards their body and decreased adoption of inflexible eating rules, which seem to explain lower levels of disordered eating behaviours. These findings seem to present empirical support for the development of intervention programs that promote a positive, affectionate and healthy relationship with one's body image, in order to prevent the inflexible adherence to eating rules and disordered eating behaviours.

Key-words: Social safeness; body appreciation; inflexible eating rules; disordered eating. 


\section{Introduction}

It is widely accepted that social safeness, i.e., feelings of social acceptance and connectedness, has its roots on early rearing interactions $[1,2]$. Research has shown that social safeness is not only related to the absence of negative rearing experiences (of threat, abuse, or neglect) but rather to the presence of positive signals (such as care, reassurance, and support) [e.g., 3]. Further, current feelings of social safeness seem to be linked with more effective emotion regulation processes (i.e., adaptive strategies through which individuals modulate their emotions to appropriately respond to internal and environmental demands) and with well-being and positive emotional states [4]. Besides, experiences of feeling safe and secure in the social world have been negatively associated with several psychopathological indicators (e.g., feelings of inferiority, shame, and submissiveness) and symptomatology (e.g., depressive and anxiety symptoms) $[5,6]$.

Empirical studies demonstrated that interpersonal contexts may have an important role in the etiology and maintenance of body and eating psychopathology [e.g., 7, 8]. Particularly, consistent theoretical and empirical accounts indicate that interpersonal sensitivities and perceptions of being in an unsafe position in the social group represent important risk pathways to the adoption of maladaptive body and eating-related behaviours $[9,10]$. In fact, eating psychopathology may result from the harmful effect of striving strategies for social approval [9-11]. In accordance with the evolutionary perspective, socialization is a fundamental need for individual survival due to the advantages of living in groups and the dangers of social isolation $[4,12$, 13]. In this line, the need to belong implies to be accepted, valued, and chosen by significant others for different roles (e.g., friend, sexual partner, or ally) [14]. In accordance with this perspective, humans have developed a set of social motivational 
systems to be attractive by others, and thus evolved to be particularly aware of the qualities valued by their social group and to strive to present attractive features, in order to be accepted and praised by others [15]. In our society, social attractiveness is highly associated with body image. Indeed, overtime the female body shape has been considered an indicator of social rank and attractiveness [16], and most women tend to overestimate physical appearance as a self and social evaluation domain [10]. However, since the decade of 1970 , the female beauty ideal changed from voluptuous and curvy to angular and thin in modern Western societies [e.g., 17]. Furthermore, nowadays, thinness is considered a characteristic valued by the social group, due its association with health, success, status, and happiness $[18,19]$. In this context, for women who perceive their weight and body shape as significantly different from the socially valued thin figure and subsequently as a source of shame, dieting may emerge as a strategy to control one's body appearance and to enhance one's status in the social group [e.g., 7, 8, 10, 20, 21].

Although dieting or restrictive eating patterns may be advisable and appropriate in certain cases (such as in obesity and diabetes), empirical evidences suggested that, in normal-weight individuals, dieting might represent a robust predictor of weight gain and eating psychopathology [20, 22]. Recent evidences indicated that, when one follows eating rules blindly, dieting may trigger disordered eating-related behaviours with significant damaging consequences [23]. Moreover, Duarte and colleagues [24] showed that an inflexible adoption of eating rules is a central process for understanding eating psychopathology in teenage girls. Indeed, the inflexible adherence of eating rules without meeting internal and external cues, has been conceptualized as a maladaptive emotional strategy to face body dissatisfaction, 
which has a paradoxical effect by increasing body and eating-related concerns and the adoption of maladaptive eating behaviours $[25,26]$.

In contrast, a growing body of literature has found that self-compassion is linked to lower levels of body dissatisfaction, body preoccupation, food-related concerns, and more adaptive eating behaviours [10, 27, 28]. A recent study by Homan and Tylka [29] highlighted the emotion regulation role of self-compassion by reporting that this process acts as an enhancer of body acceptance and appreciation.

Body appreciation characterizes a positive, accepting and respecting attitude towards body image, regardless of its appearance [30]. This appreciative attitude does not correspond to the opposite of body image dissatisfaction; in fact, body appreciation entails more than being satisfied with one's own body image and can be understood as holding a compassionate attitude towards one's own body image [31]. Body appreciation is, therefore, the ability to accept and to be kind towards perceived defects or flaws in appearance and also to recognize the unappreciated body characteristics as part of the common human experience [31]. Further, body appreciation can be conceptualized as an adaptive emotion process (i.e., as a protective and respectful attitude towards one's body) through the rejection of unrealistic ideals, which involves the acceptance of the specificities and imperfections that one's body may have [32]. Additionally, Wood-Barcalow and colleagues [32] suggested that there is a positive relationship between body appreciation and meaningful social connections and close emotional ties with family, friends, and romantic partners. Along these lines, positive and secure social relationships may be associated with a more favourable and accepting relationship with one's body which, in turn, seems to promote the engagement in healthy behaviours. Therefore, the primary aim of the current study is to test a mediator model in which it is 
hypothesized that feelings of social safeness and connectedness are negatively linked to disordered eating attitudes and behaviours, through the mechanisms of different emotion regulation processes. Particularly, we predict that the extent to which feelings of social connectedness explain lower levels of disordered eating is mediated by body appreciation and lower tendencies to adopt inflexible eating rules.

\section{Method and Materials}

\subsection{Participants}

A total of 253 Portuguese women from the general population, aged between 18 and 50 years old $(M=25.00 ; S D=7.15)$ were enrolled in the study. Participants' Body Mass Index mean was $21.76 \mathrm{Kg} / \mathrm{m}^{2}(S D=3.26)$, which corresponds to normal weight values. Twenty-seven participants $(10.67 \%)$ were underweight $(\mathrm{BMI}<18.5)$, $189(74.70 \%)$ presented a normal weight $(18.5 \geq \mathrm{BMI} \leq 25.0)$, and $37(14.63 \%)$ were overweight $(\mathrm{BMI}>25)$, which reflects the BMI distribution in the female Portuguese population [33].

\subsection{Procedures}

This study is part of a wider ongoing research regarding the role of distinct emotion regulation processes in eating psychopathology. The study's procedures respected all ethical and deontological requirements inherent to scientific research.

The study's sample was obtained through online advertisements via a social network (Facebook) and by e-mail invitations, in which was included a statement that clarified the procedure and aims of the study, and the criteria for selecting participants. The advertisements also included an Internet link which redirected potential participants to an online survey. Before answering self-report measures, all 
individuals who accepted to take part in the study were fully informed about the voluntary nature of their participation and the confidentiality of the collected data, and provided their written inform consent.

Self-report questionnaires were initially completed by 312 participants. In accordance with the aims of this study, 59 participants were excluded due to one or more of the following reasons: a) being male; b) being younger than 18 years old or older than 50 years old. This cleaning process resulted in a final sample of 253 female participants.

\subsection{Measures}

Body Mass Index. BMI was calculated through the Quetelet Index, based on participants' self-reported weight and height $\left(\mathrm{kg} / \mathrm{m}^{2}\right)$.

Social Safeness and Pleasure Scale (SSPS; [2]; Dinis, Matos, \& Pinto-Gouveia, 2008). SSPS was designed to assess positive feelings and emotions linked to experiencing one's social word as safe and soothing (e.g., "I feel content within my relationships"). Participants were asked to rate on a 5-point scale ( 1 = "Almost never" to $5=$ "Almost ever") the extent to which they agree with each of the 11 items. In the original version, SSPS revealed a very good internal consistency with a Cronbach's alpha of 0.91 [2]. In the current study the Cronbach's alpha was 0.92.

Body Appreciation Scale-2 (BAS-2; [32, 34]. BAS-2 is a 10-item self-report measure of body image appreciation, respect and acceptance (e.g., "I take a positive attitude towards my body"). Responses are rated on a 5-point scale ( $1=$ "Never" to 5 $=$ "Always"), with higher scores reflecting greater body appreciation attitudes. The scale revealed, in both the original and the Portuguese validation studies, very good 
internal consistencies, with Cronbach's alphas of 0.97 and 0.95 , respectively $[32,34]$. In the present study, BAS-2's Cronbach's alpha was 0.95.

Inflexible Eating Questionnaire (IEQ; Duarte, Ferreira, Pinto Gouveia, \& Martinho, 2016). IEQ is an 11-item scale designed to measure the engagement in inflexible and rigid eating rules (e.g., "I rather follow my eating rules than eating in function of the context or my hunger or will"). Participants are asked to select, on a 5point scale $(1=$ "I totally disagree" to $5=$ "I totally agree"), the number that best represents their agreement with each sentence. Higher scores on this scale indicate higher inflexible adherence in eating attitudes and behaviours. The scale showed a very good internal consistency scale (0.95), construct and discriminant validity, and presented a good temporal stability. A recent study, with a large sample of women from the general population, revealed that IEQ presents positive associations with body mass index (BMI), eating psychopathology, and anxiety, depressive and stress symptoms and negative associations with different adaptive emotion processes (such as psychological and body image flexibility, cognitive defusion, decentering, and selfcompassion) [35]. Concerning the current study, IEQ's Cronbach's alpha was 0.94.

Eating Disorder Examination Questionnaire (EDE-Q; [36, 37]). This is the selfreport form version of the Eating Disorder Examination (EDE; [38]), a semi structured interview which evaluates the frequency and severity of pathological eating behaviours and attitudes. EDE-Q is composed of 36 items, divided in four subscales: restraint, eating concern, weight concern and shape concern. The items are rated for frequency of occurrence (items 1-15, on a scale ranging from $0=$ "None" to $6=$ "Every day") or for severity (items 29-36, on a scale ranging from $0=$ "None" to $6=$ "Extremely"), taking into account the last 28 days. This scale has shown to be a valid and reliable instrument, with high values of internal consistency (0.94), for both the 
original [36] and the Portuguese [37] versions. The current study used the global score of the EDE-Q (calculated by the average of the EDE-Q's subscales), which presented an alpha value of 0.94 .

\subsection{Data analysis}

The software IBM SPSS Statistics (version 23.0; IBM; Chicago, IL) was used to conduct descriptive and correlational analyses. Pearson product-moment correlation analyses were performed to explore the relationship between BMI, social safeness (SSPS), body appreciation (BAS-2), inflexible eating (IEQ) and disordered eating (EDE-Q). Coefficients were analysed considering Cohen's guidelines [39].

Furthermore, to calculate the presumed relationships between study variables a series of path analyses were conducted with the software "Analysis of Momentary Structure" (AMOS; [40]). These analyses aimed to examine whether the link between current feelings of social belonging and safeness (exogenous variable) and disordered eating (endogenous variable) was mediated by body appreciation and inflexible eating (endogenous mediator variables). In this model, BMI was considered a confounding variable and its effect was controlled for.

The plausibility of the model was examined through a set of goodness-of-fit indices: The chi-square goodness-of-fit (which reveals a good adjustment when nonsignificant; [41]), the Comparative Fit Index (CFI) and the Tucker and Lewis Index (TLI) which present a good model fit when values are superior to 0.95 [42]. The Root Mean Squared Error of Approximation (RMSEA, which represents an excellent fit when values are inferior to 0.08 [42]) was also used. 
The significance of the mediational paths was examined via the Bootstrap resampling method with 5000 samples and $95 \%$ bias-corrected confidence intervals (CI) around the standardized estimates of total, direct and indirect effects. The effects are considered significant when zero is not included within the CI [43].

\section{Results}

\subsection{Preliminary analyses}

The coefficients of skewness and kurtosis, indicated that in the current sample there was no violation of normal distribution, with skewness values (Sk) ranging from - 0.02 to 1.38 (in the IEQ and in the EDE-Q, respectively), while the kurtosis values $(\mathrm{Ku})$ ranged from 0.06 to 1.97 (in the BAS-2 and the EDE-Q, respectively) [43]. Furthermore, the data was shown to be suitable, as indicated by preliminary analyses, which pointed to normality, linearity, homoscedasticity, independence of errors, and to singularity and absence of multicollinearity among the variables [44].

\subsection{Descriptive statistics and correlations analyses}

Descriptive statistics for the study variables are presented in Table 1 . The means and standard deviations of the variables in study were similar to those obtained in previous studies with nonclinical female samples.

Results from correlation analyses revealed that BMI presented negative correlations with social safeness (SSPS) and body appreciation (BAS-2), with weak and moderate magnitudes, respectively. On the other hand, BMI was revealed to be positively associated with inflexible eating (IEQ) and with the global score of EDE-Q. In turn, a positive and moderate correlation was found between feelings of social safeness and body appreciation. In contrast, the relationship of social safeness and 
inflexible eating and EDE-Q were negative. Also, body appreciation was negatively correlated with both inflexible eating and EDE-Q, with moderate and strong magnitudes respectively. Finally, a strong and positive correlation was observed between IEQ and EDE-Q.

\section{Please insert Table 1 here}

\subsection{Path analysis}

First, a fully saturated model (i.e., with zero degrees of freedom) comprising 20 parameters was tested to explore the potential role of these variables in the path model. The model explained $28 \%$ of body appreciation, $18 \%$ of inflexible eating and $58 \%$ of EDE-Q's variance. In this model, two paths were not significant: the direct effect of social safeness on EDE-Q ( $\left.b_{\mathrm{SSPS}}=0.01 ; \mathrm{SE}_{\mathrm{b}}=0.21 ; Z=0.50 ; p=0.96\right)$ and the direct effect of social safeness on inflexible eating $\left(b_{\mathrm{SSPS}}=-0.05 ; \mathrm{SE}_{\mathrm{b}}=0.10 ; Z=-\right.$ $0.53 ; p=0.59)$. Concerning these results, these paths were eliminated and the model was recalculated.

The readjusted model revealed that all path coefficients were statistically significant $(p<0.001)$ and presented an excellent model fit, with a non-significant chi-square $\left[\chi_{(2)}^{2}=2.99, p=0.87\right]$ and excellent goodness-of-fit indices $(\mathrm{CFI}=1.00$; $\mathrm{TLI}=1.02 ; \mathrm{RMSEA}=.0 .00,[\mathrm{IC}=0.00-0.07 ; p=0.93])$.

Results indicated that social safeness accounted for $25 \%$ of body appreciation. Additionally, $18 \%$ of inflexible eating was explained by its indirect effect through body appreciation, and 58\% of EDE-Q was explained by its indirect effect through body appreciation and inflexible eating. 
Specifically, social safeness presented a direct effect of $0.35\left(b_{\mathrm{SSPS}}=0.38 ; \mathrm{SE}_{\mathrm{b}}=\right.$ 0.06; $Z=6.39 ; p<0.001)$ on body appreciation. In turn, body appreciation presented direct effects of $-0.35\left(b_{\text {BAS_2 }}=-0.50 ; \mathrm{SE}_{\mathrm{b}}=0.09 ; Z=-5.73 ; p<0.001\right)$ and -0.32

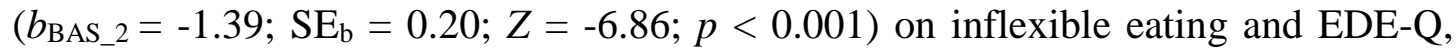
respectively. Finally, inflexible eating showed a direct effect on EDE-Q of 0.48 ( $b_{\mathrm{IEQ}}$ $\left.=1.46 ; \mathrm{SE}_{\mathrm{b}}=0.14 ; Z=10.71 ; p<0.001\right)$.

The analysis of indirect effects allowed to observe that social safeness presented an indirect effect on EDE-Q, of -0.17 (95\% CI -0.24 to -0.11 ), mediated by body appreciation and inflexible eating. Furthermore, it was verified that social safeness revealed an indirect effect of -0.12 (95\% CI -0.18 to -0.07$)$ on inflexible eating, through the effect of body appreciation. Finally, body appreciation showed an indirect effect of -0.17 (95\% CI -0.23 to -0.11) on EDE-Q, through inflexible eating.

To sum up, these findings seem to reveal that the impact of feelings of social safeness on eating psychopathology severity was carried by the mechanisms of body appreciation and inflexible eating.

Please insert Figure 1 around here

\section{Discussion}

The current study aimed at testing a model which explores the mediator role of body appreciation and inflexible eating rules in the relationship between social safeness and disordered eating, controlling for BMI, in a sample of women from the general population.

Findings corroborated that social safeness is positively linked with adaptive emotion regulation processes and negatively associated with ill-being indicators $[4,5$, 
6]. Particularly, results revealed that current feelings of social acceptance and connectedness are positively associated with a kind and respectful attitude towards one's body, even when one is not totally pleased with all its aspects (body appreciation). This result seems to corroborate the positive link between meaningful connections and close emotional ties and the development of body appreciation, reported by Wood-Barcalow and colleagues [32]. Further, our results extend previous literature by revealing that feelings of belonging and social safeness are negatively linked to the adoption of inflexible or rigid eating rules and disordered eating attitudes and behaviours. Also, results demonstrated a significant negative association between body appreciation and, both, inflexible eating rules and a global indicator of eating psychopathology (as assessed by EDE-Q). Finally, current findings supported previous empirical accounts [e.g., 23, 24] by demonstrating a strong and positive correlation between an inflexible adherence to eating rules (i.e., without meeting internal and external cues) and the adoption of maladaptive eating behaviours. Overall, these findings point out the relevant associations between these variables and, more specifically, to how interpersonal-related experiences and feelings may be related to body appreciation, the engagement in rigid eating rules, and disordered eating. To further explore these associations, path analyses were performed aiming to analyse the mediational role of body appreciation and inflexible eating rules in the association between social safeness and disordered eating, while controlling for BMI.

Results revealed that the tested model presented an excellent fit to the empirical data and explained $58 \%$ of eating psychopathology's variance. Moreover, results indicated that, in the presence of body appreciation and inflexible eating, social safeness did not directly predict disordered eating. Specifically, this model suggested that social safeness holds a significant effect on eating psychopathology, through the 
mechanisms of body appreciation and inflexible eating rules. In particular, results suggested that women who present higher levels of social safeness are more willing to reveal a positive and respectful attitude towards body image, regardless of its appearance, and decreased adoption of inflexible eating rules. In turn these mechanisms seem to explain lower levels of disordered eating.

Nevertheless, this innovative study presents some limitations. Firstly, the crosssectional nature of the study does not allow conclusions regarding causality. In this way, it would be interesting to conduct this study using a longitudinal design. The second limitation of the present study is related to the multidetermination of eating psychopathology, which makes the tested model a limited one, due to the fact that other variables can be involved in the examined associations. However, since the main aim of the present study was to specifically explore the relationship between social safeness and disordered eating attitudes and behaviours, and the mediator effect of body appreciation and inflexible eating rules on this link, other relevant variables were beyond this study's scope and were not examined. Upcoming studies should then expand this model considering other variables that may contribute to the complex phenomenon of eating psychopathology. Additionally, given that this study comprised a sample exclusively composed of women from the general population, the found data may not be generalized. Hence, future research with different samples (such as male and clinical samples) is required. However, the choice of only including women in this study was due to the fact that body and eating-related difficulties are much more common in women rather than in men [45]. Finally, the exclusive use of self-report measures can also represent a limitation of this study. Future studies should use other types of measures (e.g., interviews), in order to confirm the obtained results. 
Nonetheless, this study offers important insights for research on body image and eating-related difficulties. In fact, this study suggested that women who perceive their social world as safe seem to develop a more favourable and accepting relationship towards their body and its perceived defects or flaws in their appearance (instead of being harshly self-critical), which seems to decrease the inflexible and rigid subscription to eating rules and the engagement in unhealthy eating behaviours. On the other hand, the current study seems to demonstrate that the perceptions of being in an unsafe position in relation to one's social group represent risk pathways to the adoption of maladaptive body and eating-related behaviours $[9,10]$. In fact, these data are in line with previous literature [8] suggesting that when a woman does not feel safe in social contexts and perceives others as unsafe or threatening, she may feel the need to stay vigilant and be ready to engage in defensive responses and strive for social acceptance. In this line, the inflexible adherence to eating rules may emerge as a strategy to control one's body appearance and to enhance one's social status in the social group.

The current study offers important directions for the development of preventive and therapeutic intervention programmes focused on body image and eating behaviours. Indeed, this study evidences the importance of promoting a positive and respectful attitude towards the body, which seems an important mechanism to decrease the engagement in inflexible eating rules and disordered eating behaviours.

\section{Compliance with ethical standards}

Ethical approval: All procedures performed in studies involving human participants were in accordance with the ethical standards of the institutional research committee 
and with the 1964 Helsinki declaration and its later amendments or comparable ethical standards.

Informed consent: Informed consent was obtained from all individual participants included in the study.

Conflict of interest: The authors of this manuscript declare no conflict of interest.

\section{References}

1. Gilbert P (2010) Compassion focused therapy: Distinctive features. London. Routledge.

2. Gilbert P, McEwan K, Mitra R, Richter A, Franks L, Mills A, Bellew R, Gale C (2009) An exploration of different types of positive affect in students and in patients with bipolar disorders. Clin Neuropsychiatry 6(4): 135-143.

3. Richter A, Gilbert P, McEwan K (2009) Development of an early memories of warmth and safeness scale and its relationship to psychopathology. Psychol Psychother 82:171-184. doi:10.1348/147608308X395213.

4. Gilbert P (2005) Compassion and cruelty: a biopsychosocial approach. In: Gilbert P (ed) Compassion: conceptualisation, research and use in psychotherapy. Routledge, London, pp 9-74.

5. Gilbert P, McEwan K, Mitra R, Franks L, Richter A, Rockliff H (2008) Feeling safe and content: A specific affect regulation system? Relationship to depression, anxiety, stress, and self-criticism. The Journal of Positive Psychology 3(3): 182-191. doi: 10.1080/17439760801999461

6. Kelly A, Zuroff D, Leybman M, Gilbert P (2012) Social Safeness, Received Social Support, and Maladjustment: Testing a Tripartite Model of Affect Regulation. Cognitive Therapy and Research 36(6): 815-826. doi 10.1007/s10608-011-9432-5. 
7. Goss K, Gilbert P (2002) Eating disorders, shame and pride: A cognitivebehavioural functional analysis. In: Gilbert P, Miles J (eds) Body shame: Conceptualization, research \& treatment. Brunner-Routledge, Hove, UK, pp $219-255$.

8. Pinto-Gouveia J, Ferreira C, Duarte C (2014) Thinness in the pursuit for social safeness: an integrative model of social rank mentality to explain eating psychopathology. Clin Psychol Psychother 21(2):154-165. doi:10.1002/cpp.1820.

9. Bellew R, Gilbert P, Mills A, McEwan K, Gale C (2006) Eating attitudes and striving to avoid inferiority. Eat Disord 14(4):313-322. doi:10.1080/10640260600796242.

10. Ferreira C, Pinto-Gouveia J, Duarte C (2013) Physical appearance as a measure of social ranking: the role of a new scale to understand the relationship between weight and dieting. Clin Psychol Psychother 20(1):5566. doi:10.1002/cpp.769.

11. Duarte C, Ferreira C, Pinto-Gouveia J (2016) At the core of eating disorders: Overvaluation, social rank, self-criticism and shame in anorexia, bulimia and binge eating disorder. Comprehensive Psychiatry 66: 123-131. doi: 10.1016/j.comppsych.2016.01.003.

12. Baumeister R, Leary M (1995) The need to belong: Desire for interpersonal attachments as a fundamental human motivation. Psychol Bull 117(3):497529. doi:10.1037/0033-2909.117.3.497.

13. Choenarom C, Williams R, Hagerty B (2005) The Role of Sense of Belonging and Social Support on Stress and Depression in Individuals With 
$\begin{array}{llll}\text { Depression. } & \text { Arch } & \text { Psychiatr } & \text { Nurs }\end{array}$ doi:10.1016/j.apnu.2004.11.003.

14. Gilbert P (2007) The evolution of shame as a marker for relationship security: a biopsychosocial approach. In: Tracy J, Robin R, Tangney J (eds) The selfconscious emotions: theory and research. Guilford, New York, pp 283-309.

15. Allan S, Gilbert P (1995) A social comparison rating scale: psychometric properties and relationship to psychopathology. Pers Individ Dif 19:293-299. doi:10.1016/0191-8869(95)00086-L.

16. Gatward N (2007) Anorexia nervosa: An evolutionary puzzle. European Eating Disorders Review 15: 1-12. doi: 10.1002/erv.718.

17. Garner M, Garfinkel P, Schwartz D, Thompson M (1980) Cultural expectations of thinness in women. Psychol Rep 47(2):483-491. doi:10.2466/pro.1980.47.2.483.

18. Sypeck M, Gray J, Etu S, Ahrens A, Mosimann J, Wiseman C (2006) Cultural representations of thinness in women, redux: Playboy magazine's depictions of beauty from 1979 to 1999 . Body Image 3:229-235. doi:10.1016/j.bodyim.2006.07.001.

19. Buote V, Wilson A, Strahan E, Gazzola, S, Papps F (2011) Setting the bar: sociocultural norms for women's and men's ideal appearance in real-world contexts. Body Image 8(4):322-334. doi:10.1016/j.bodyim.2011.06.002.

20. Brown A, Parman K, Rudat D, Craighead L (2012) Disordered eating, perfectionism, and food rules. Eat Behav 13(3):47-353. doi:10.1016/j.eatbeh.2012.05.011.

21. Goss K, Allan S (2009) Shame, pride and eating disorders. Clin Psychol Psychother 16(4): 303-316. doi:10.1002/cpp.627. 
22. Holmes M, Fuller-Tyszkiewicz M, Skouteris H, Broadbent J (2015) Understanding the link between body image and binge eating: a model comparison approach. Eating and Weight Disorders 20(1): 81-89. doi:10.1007/s40519-014-0141-4

23. Ferreira C, Trindade I A, Martinho A (2016) Explaining rigid dieting in normal-weight women: the key role of body image inflexibility. Eating and Weight Disorders 21(1): 49-51. doi:10.1007/s40519-015-0188-X

24. Duarte C, Ferreira C, Trindade A, Pinto-Gouveia J (2015) Normative body dissatisfaction and eating psychopathology in teenage girls: the impact of inflexible eating rules. Eat Weight Disord 21(1):41-48. doi:10.1007/s40519015-0212-1.

25. Mann T, Ward A (2001) Forbidden fruit: does thinking about a prohibited food lead to its consumption? International Journal of Eating Disorders 29(3): 319-327. doi:10.1002/eat.1025.

26. Stice E, Marti C, Durant S (2011) Risk factors for onset of eating disorders: evidence of multiple risk pathways from an 8-year prospective study. Behav Res Ther 49(10):622-627. doi:10.1016/j.brat.2011.06.009.

27. Kelly AC, Vimalakanthan K, Carter JC (2014) Understanding the roles of self-esteem, self-compassion, and fear of self-compassion in eating disorder pathology: An examination of female students and eating disorder patients. Eating Behaviors 15(3): 388 -391. doi: 10.1016/j.eatbeh.2014.04.008.

28. Taylor M, Daiss S, Krietsch K (2015) Associations among self-compassion, mindful eating, eating disorder symptomatology, and body mass index in college students. Translational Issues in Psychological Science 1(3): 229-238. doi: $10.1037 / \operatorname{tps} 0000035$. 
29. Homan KJ, Tylka TL (2015) Self-compassion moderates body comparison and appearance self-worth's inverse relationships with body appreciation. Body Image 15: 1-7. doi: 10.1016/j.bodyim.2015.04.007.

30. Avalos L, Tylka T L, Wood-Barcalow N (2005) The Body Appreciation Scale: development and psychometric evaluation. Body Image 2(3): 285-97. doi: 10.1016/j.bodyim.2005.06.002.

31. Tylka T, Wood-Barcalow, N (2015) The Body Appreciation Scale-2: Item refinement and psychometric evaluation. Body Image 12:53-67. doi:10.1016/j.bodyim.2014.09.006.

32. Wood-Barcalow N, Tylka T, Augustus-Horvath C (2010) But I like my body: Positive body image characteristics and a holistic model for young adult women. Body Image 7:106-116. doi:10.1016/j.bodyim.2010.01.001.

33. Poínhos R, Franchini B, Afonso C, Correia F, Teixeira VH, Moreira P, Durão C, Pinho O, Silva D, Lima Reis JP, Veríssimo T, de Almeida MDV (2009) Alimentação e estilos de vida da população Portuguesa: metodologia e resultados preliminares [Alimentation and life styles of the Portuguese population: methodology and preliminary results]. Alimentação Humana 15(3):43-60.

34. Marta-Simões J, Mendes L, Trindade I, Ferreira C.(2016) Validation of the Body Appreciation Scale-2 for Portuguese women. Poster presented at the 3rd IPLeiria’s International Health Congress, Leiria, Portugal.

35. Duarte C, Ferreira C, Trindade I, Pinto-Gouveia J. (2015, September). The backlash of inflexible eating rules: Development and validation of a new measure of eating-related inflexibility. Poster session presented at the meeting of the 45th EABCT Congress, Jerusalem, Israel. 
36. Fairburn C, Beglin S (1994) Assessment of eating disorders: interview or self-report questionnaire? Int J Eat Disord 16:363-370. doi:10.1002/1098108X(199412)16:4<363::AID-EAT2260160405>3.0.CO;2-\#.

37. Machado P, Martins C, Vaz A, Conceição E, Bastos A, Gonçalves S (2014) Eating disorder examination questionnaire: psychometric properties and norms for the Portuguese population. Eur Eat Disord Rev 22(6):448-453. doi:10.1002/erv.2318.

38. Cooper Z, Cooper P, Fairburn C (1989) The validity of the eating disorder examination and its subscales. $\mathrm{Br} \quad \mathrm{J}$ Psychiatry 154(6):807-812. doi:10.1192/bjp.154.6.807.

39. Cohen J, Cohen P, West S, Aiken L (2003) Applied multiple regression/correlation analysis for the behavioral sciences, 3ed. Hillsdale: Erlbaum.

40. Arbuckle J (2006) Amos (Version 7.0) [Computer program]. Chicago: SPSS.

41. Hair JF, Anderson RE, Tatham RL, Black WC (1998) Multivariate Data Analysis, (5th Edition). Upper Saddle River, NJ: Prentice Hall.

42. Hooper D, Coughlan J, Mullen M (2008) Structural Equation Modelling: Guidelines for Determining Model Fit. Electronic Journal of Business Research Methods 6(1): 53-60.

43. Kline RB (2005). Principles and Practice of Structural Equation Modeling, 2ed. New York: The Guilford Press.

44. Field A (2004) Discovering statistics using SPSS, 3ed. London: Sage Publications.

45. Striegel-Moore RH, Rosselli F, Perrin N, DeBar L, Wilson GT Alexis May A, Kraemer HC (2009) Gender Difference in the Prevalence of Eating 
Disorder Symptoms. Int J Eat Disord. 42(5): 471-474. doi: 10.1002/eat.20625. 
Table 1

Cronbach's Alphas ( $\alpha$ ), Means (M), Standard Deviations (SD), and Intercorrelation scores on self-report measures $(N=253)$

\begin{tabular}{lcccccc}
\hline Measures & $\boldsymbol{M}$ & $\boldsymbol{S D}$ & $\mathbf{1}$ & $\mathbf{2}$ & $\mathbf{3}$ & $\mathbf{4}$ \\
\hline 1. BMI & 21.76 & 3.26 & 1 & & & \\
2. SSPS & 32.42 & 6.91 & $-0.17^{* *}$ & 1 & & \\
3. BAS_2 & 27.87 & 7.45 & $-0.36^{* * *}$ & $0.41^{* * *}$ & 1 & \\
4. IEQ & 16.00 & 10.67 & $0.26^{* * *}$ & $-0.19^{* *}$ & $-0.40^{* * *}$ & 1 \\
5. EDE_Q & 1.26 & 1.13 & $0.42^{* * *}$ & $-0.25^{* * *}$ & $-0.58^{* * *}$ & $0.66^{* * *}$ \\
\hline
\end{tabular}

Note $:$ BMI $=$ Body Mass Index SSPS $=$ Social Safeness and Pleasure Scale; BAS_2 = Body Appreciation Scale_2; IEQ = Inflexible Eating Questionnaire; EDE_Q = Eating Disorder Examination Questionnaire.

$* * * p<0.001 ; * * p<0.010 ; * \mathrm{p}<0.05$ 
Figure. 1. Final path model

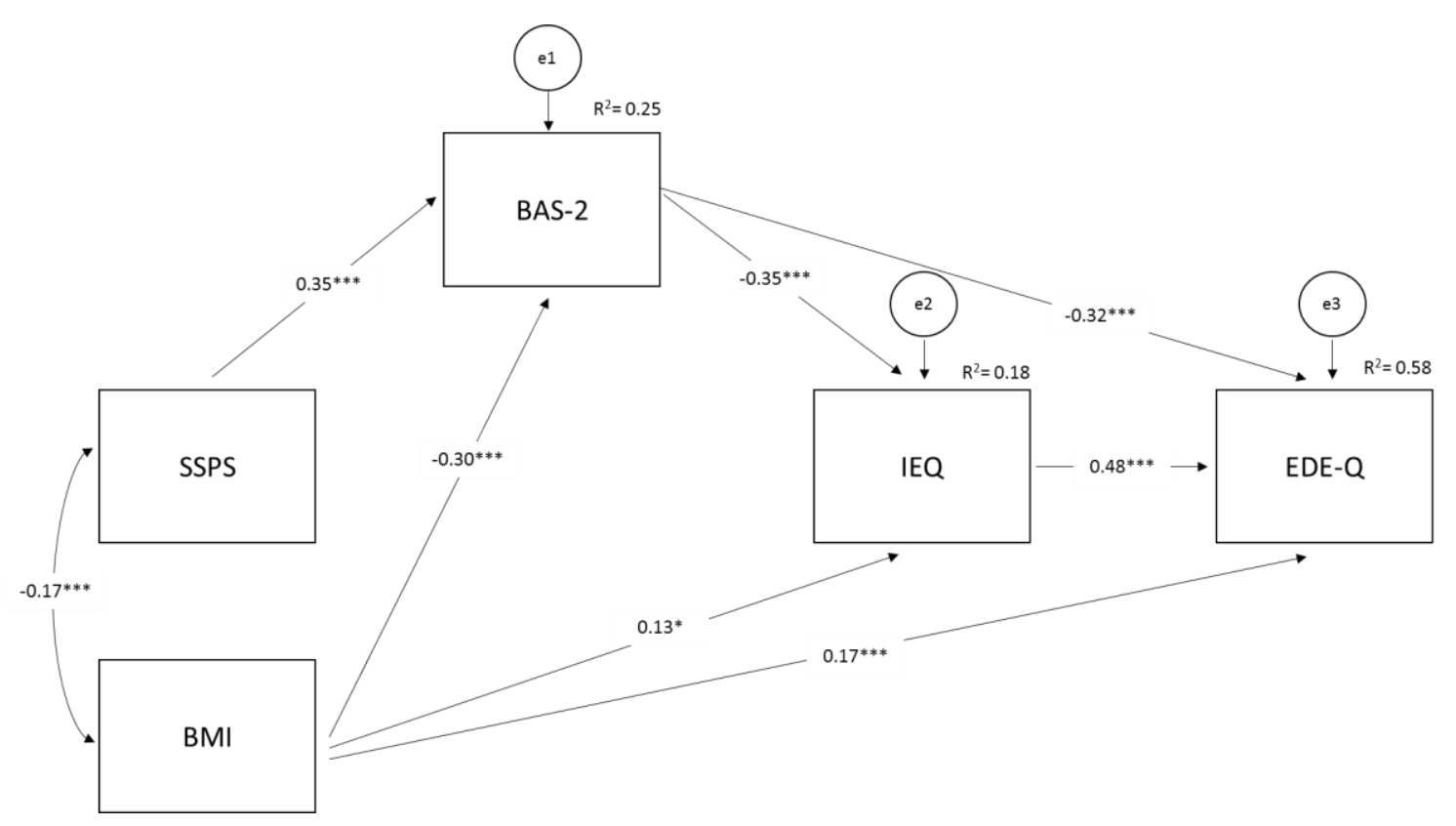

Note: Path model showing the association between social safeness (SSPS) and disordered eating (EDE-Q), mediated by body appreciation (BAS-2) and inflexible eating rules (IEQ), controlling for BMI effect, estimates and square multiple correlations $\left(R^{2}\right)(N=253)$.

All path coefficients are significant at the 0.05 level; *p $<0.05 ; * * * \mathrm{p}<.0 .001$; 